\title{
Distribution of Gastropods in the Intertidal Environment of South, Middle and North Andaman Islands, India
}

\author{
Chinnusamy Jeeva, P. M. Mohan, K. K. Dil Baseer Sabith, Vibha V. Ubare, \\ Mariyappan Muruganantham, Radha Karuna Kumari
}

Department of Ocean Studies and Marine Biology, Pondicherry University, Brookshabad Campus, Port Blair, India

Email: jeevasanthose@gmail.com,pmmtu@yahoo.com, dilbaseersabith@gmail.com, vibhaubare@gmail.com, vmmuruga@gmail.com,krnkumari13@gmail.com

How to cite this paper: Jeeva, C., Mohan, P.M., Sabith, K.K.D.B., Ubare, V.V., Muruganantham, M. and Kumari, R.K. (2018) Distribution of Gastropods in the Intertidal Environment of South, Middle and North Andaman Islands, India. Open Journal of Marine Science, 8, 173-195.

https://doi.org/10.4236/ojms.2018.81009

Received: December 25, 2017

Accepted: January 28, 2018

Published: January 31, 2018

Copyright $\odot 2018$ by authors and Scientific Research Publishing Inc. This work is licensed under the Creative Commons Attribution International License (CC BY 4.0).

http://creativecommons.org/licenses/by/4.0/

(c) (i) Open Access

\begin{abstract}
Andaman and Nicobar Islands, the gastropod diversity is high, due to the majority of shores are rocky. The wet rocky shore promotes algal growth, which is ultimate for feeding ground for gastropod growth and development leading to more diversity. The global warming, anthropogenic activities, industrial and domestic pollution, etc., have accelerated the loss of coastal and marine biodiversity components over the last few decades which has been of great concern. However, except global warming, the other factors were of least concern with reference to Andaman and Nicobar Islands biodiversity due to a pristine environment. Therefore, exploration of biodiversity in these islands is essential to create a baseline data for record and future research. Four locations of south to north Andaman Islands which represented Carbyns Cove from south Andaman, Rangat and Mayabunder from the Middle Andaman and Diglipur from the North Andaman were selected for this study. Gastropoda species were collected for a period of one year in three prevailing seasons of Andaman and Nicobar Islands. They are South West Monsoon (SW monsoon), North East Monsoon (NE Monsoon) and Non Rainy Seasons (NR Seasons). The present study of gastropods distribution in the South, Middle and North Andaman groups of Islands suggested that there are 71 species belonging to 52 genus and 33 families. At any one of the time and any one of the locations, only one occurrence was noticed for 38 species and remaining 33 species were overlapping with respect to stations and seasons. Evaluation of the cluster suggested that Cluster A (NR season in Carbyns Cove) and Cluster B (NE and SW Monsoon in Mayabunder) as a separate entity among the 12 combinations of stations and seasons due to their species representation. It
\end{abstract}


was also concluded that out of 71 studied species, 3 species were not reported till date in these Island environment and 2 species from any other parts of India.

\section{Keywords}

Gastropoda, Abundance, Species Composition, Andaman Sea, Bay of Bengal

\section{Introduction}

Andaman and Nicobar archipelago located in the central part of the Bay of Bengal consists of 572 islands. Southern tip of this archipelago is located just at 145 NM from the northern side of Indonesia and 750 NM distance from the eastern coast of India. This island is scarcely populated than the mainland India. This pristine environment supports establishment of well diversified coral communities. This archipelago is one among the 12 mega-biodiversities and also one among the 25 hotspots of the rich and highly endangered eco-regions of the world. Among the Asian countries, India is the only one country that has a long record of inventories of coastal and marine biodiversity dating back to at least two centuries.

The research history of malacological studies in India is among the oldest one which was initiated by the Asiatic Society of Bengal during the year 1784 and made a repository in the Indian Museum, Calcutta in the year 1814. Benson published a total of 90 research papers which was mainly concerned land and freshwater mollusks of the Indian subcontinent between 1830-1865 [1]. Till date, 3370 species of marine mollusks have been recorded in India, out of which 1282 species are from Andaman and Nicobar Islands [2]. The majority of mollusks are present in the marine biotopes and they occur from the backwater zone, mangroves, intertidal and shelf in deeper waters. Mollusks have been a favorite candidate for the research in the early days, especially those available on rocky shores and inter-tidal zones as they are plentiful of animal, moderately slow moving and easy to identify [3] [4] [5]. They comprise an important component of intertidal fauna and flora [6].

Among the mollusks, gastropods are more important in the coastal populations' economy, i.e. as a shell-craft industry. Over and above, the marine gastropod resources in India consisted of different types of species which are exploited regularly for various purposes like drugs, ornamental purposes as well as for their meat. Andaman and Nicobar Islands, the gastropod diversity are high, due to the majority of shores are rocky. The wet rocky shore promotes algal growth, which is ultimate for feeding ground for gastropod growth and development leading to more diversity. Further, the mangrove environment also provides ideal conditions for the productivity of gastropods [7].

The global warming, anthropogenic activities, industrial and domestic pollution, 
etc., have accelerated the loss of coastal and marine biodiversity components over the last few decades which has been of great concern. However, except global warming, the other factors were of least concern with reference to Andaman and Nicobar Islands biodiversity due to a pristine environment. Therefore, exploration of biodiversity in these islands is essential to create a baseline data for record and future research. In this regard, gastropods are a significant indicator of environmental changes, hence, an attempt had been made to understand a comprehensive gastropod diversity by the way of studies on selected locations of the south to north Andaman coast lines.

\section{Materials and Methods}

\subsection{The Study Area}

The gastropod diversity of the Andaman Islands was studied in four locations of south to north Andaman Islands which represented Carbyns Cove from south Andaman, Rangat and Mayabunder from the Middle Andaman and Diglipur from the North Andaman (Figure 1). The station descriptions are as follows:

\subsubsection{Carbyns Cove}

This station is located in the south Andaman. This coast has a length of one $\mathrm{km}$ out of that, the central part which extends to around $300 \mathrm{~m}$ is covered with sand and remaining part are exposed as rock terrain. Near the southern part of the sandy beach environment a freshwater inlet is present through which inflow of rainwater occurs during the monsoon. However, during the high tide the whole beach is submerged with sea water.

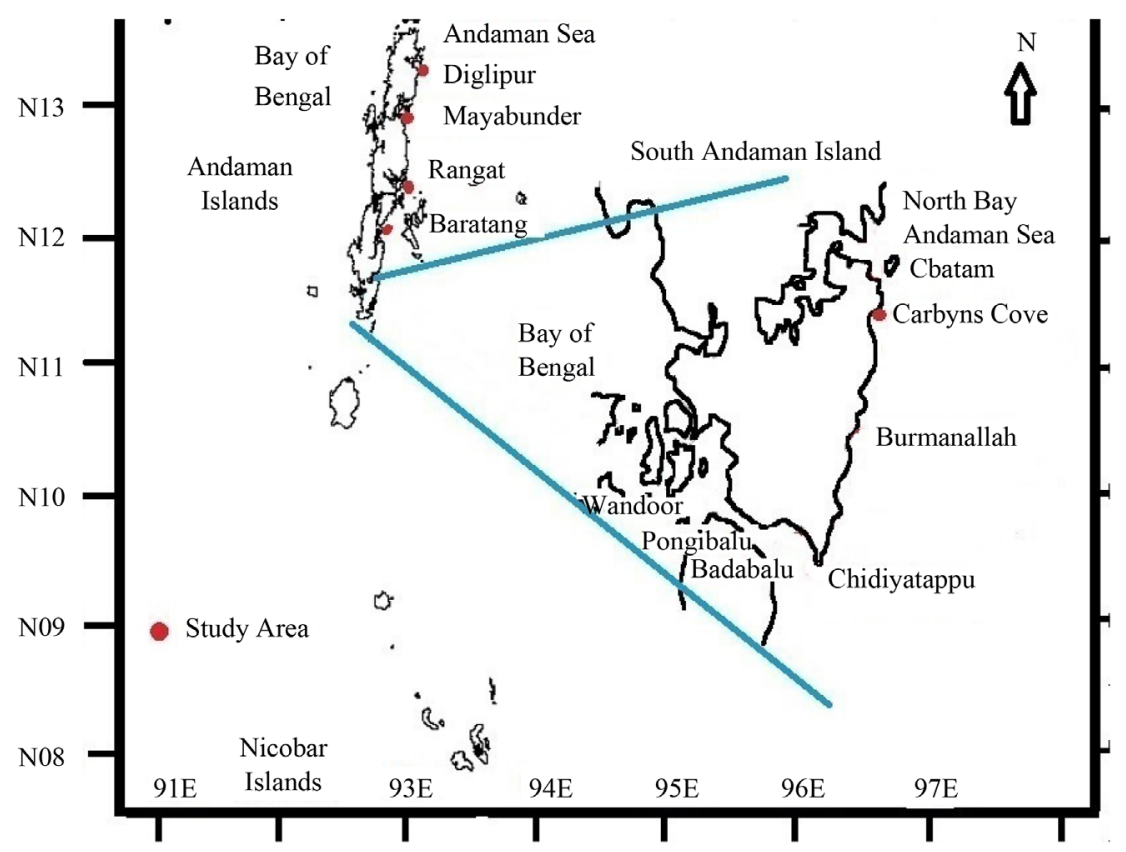

Figure 1. Study area. 


\subsubsection{Rangat}

This location is present in the southern part of Middle Andaman. This area covered a length of one $\mathrm{km}$. This site is covered by rocky outcrops with boulders and pebbles which are in many ways similar to the Carbyns Cove, however, it has a less sandy beach of around $100 \mathrm{~m}$ long with a freshwater inlet in south side. However, during the non rainy season this inlet would be blocked with boulders and pebbles while during the rainy season it opens for the freshwater flow.

\subsubsection{Mayabunder}

This station is located in the northern part of Middle Andaman. The exact location is near the eastern side of the fishery jetty. However, this area largely consists of exposed rocks with boulders and pebbles surrounded by mangrove vegetation.

\subsubsection{Diglipur}

This study area is located in the northern part of Andaman. The samples were collected at Kalipur beach. Sandy beach precedes an exposed coral basement on the eastern side while the western side has a plain and wider sand cover without any coral basement exposure along with a freshwater inlet. The high tide covers this coral basement along with the upper sandy beach. During the low tide more than a 600 to $700 \mathrm{~m}$ wide coral basement with gentle slope would be exposed.

\subsection{Methods}

All the above stations, once in four months, i.e. from August 2013 to August 2014 , systematic samples were collected throughout the coastline. The seasons of Andaman considered as a unique one, eight months, having continuous rain and four month considered as the non rainy season. Overall annual rainfall is 3394 mm. The period January to April considered Non Rainy Season (NRS). The month May to August considered as South West Monsoon (SWM) and September to December considered as North East Monsoon (NEM). So, these collections were made at the end of each season, i.e. the Second Half of December to First Half of January is representing the end of NE monsoon. The Second Half of the April to First Half of May considered as the end of the Non Rainy season and Second Half of the July to First Half of August represented as the end of the SW monsoon period. The collection of samples was within one square meter transect at two sites in each station by hand picking as mentioned by Arumugam et al. [8]. The collected animals were photographed in the field itself for its original colour and then preserved in $10 \%$ formalin for further analysis. Identification was carried out using the different authors key such as Pinn [9], Apte [10] [11], Jereb and Roper [12], Ramkrishna and Dey [13], Rao [14], D’Souza [15] and WoRMS [16] based on the colour pattern of the shell, shape, number of whorls, operculum, etc. Then identified gastropods were counted and averaged in two sites of each station and recorded for the diversity and density studies. The data for the gastropods and its diversity were presented in Table 1 . 
Table 1. Percentage Distribution of the Gastropoda Species in Different Locations and Seasons of the Present Studied Samples NER-NE Monsoon Rangat; NRR-Non Rainy Season Rangat; SWR-SW Monsoon Rangat; NEM-NE Monsoon Mayabunder; NRM-Non Rainy Season Mayabunder; SWM-SW Monsoon Mayabunder; NED-NE Monsoon Diglipur; NRD-Non Rainy Season Diglipur; SWR-SW Monsoon Diglipur; NEC-NE Monsoon Carbyns Cove; NRC-Non Rainy Season Carbyns Cove; SWC-SW Monsoon Carbyns Cove.

\begin{tabular}{|c|c|c|c|c|c|c|c|c|c|c|c|c|}
\hline Species Name & $\begin{array}{l}\text { NER } \\
2013\end{array}$ & $\begin{array}{l}\text { NRR } \\
2014\end{array}$ & $\begin{array}{l}\text { SWR } \\
2014\end{array}$ & $\begin{array}{l}\text { NEM } \\
2013\end{array}$ & $\begin{array}{l}\text { I NRM } \\
2014\end{array}$ & $\begin{array}{l}\text { SWM } \\
2014\end{array}$ & $\begin{array}{l}\text { I NED } \\
2013\end{array}$ & $\begin{array}{l}\text { NRD } \\
2014\end{array}$ & $\begin{array}{l}\text { SWD } \\
2014\end{array}$ & $\begin{array}{l}\text { NEC } \\
2013\end{array}$ & $\begin{array}{l}\text { NRC } \\
2014\end{array}$ & $\begin{array}{l}\text { SWC } \\
2014\end{array}$ \\
\hline Trochus radiates & 5.32 & --- & 1.53 & --- & --- & 2.77 & --- & --- & --- & 5.00 & --- & --- \\
\hline Monodonta australis & 1.33 & --- & --- & --- & --- & 8.33 & --- & 4.54 & 4.16 & 7.50 & --- & --- \\
\hline Tectus pyramis & ---- & --- & --- & --- & 10.52 & --- & --- & --- & 4.16 & --- & --- & --- \\
\hline Angaria delphinus & --- & --- & --- & 7.14 & --- & --- & --- & --- & --- & --- & --- & --- \\
\hline Turbo bruneus & 2.66 & --- & --- & --- & --- & 2.77 & 2.85 & 4.54 & 12.50 & 2.50 & --- & --- \\
\hline Lunella cinerea & 1.33 & --- & --- & --- & --- & --- & --- & --- & --- & --- & --- & --- \\
\hline Turbo sp. & --- & --- & --- & --- & --- & --- & --- & --- & --- & --- & 33.33 & --- \\
\hline Turbonilla felicita & --- & --- & --- & --- & --- & --- & --- & --- & --- & 2.5 & -- & --- \\
\hline Nerita albicilla & 5.33 & 88.23 & 10.76 & --- & 5.26 & 8.33 & 17.14 & 13.63 & 20.83 & 25.00 & --- & 52.62 \\
\hline Nerita oryzarum & 1.33 & --- & 7.68 & --- & 36.84 & --- & 2.85 & --- & 8.32 & 2.50 & --- & --- \\
\hline Nerita polita & 16.00 & --- & 6.15 & --- & 5.26 & --- & --- & 9.09 & --- & --- & --- & --- \\
\hline Nerita histrio & --- & --- & --- & --- & --- & --- & 14.28 & -- & --- & --- & --- & --- \\
\hline Nerita chamaeleon & -- & --- & -- & -- & 10.52 & 5.55 & 2.85 & --- & -- & -- & -- & --- \\
\hline Nerita insculpta & --- & --- & 15.38 & --- & --- & --- & --- & --- & --- & --- & --- & --- \\
\hline Nerita costata & --- & --- & 13.84 & --- & --- & --- & --- & --- & --- & --- & --- & 15.78 \\
\hline Cellana radiata & 1.33 & --- & --- & --- & --- & --- & --- & --- & --- & --- & --- & --- \\
\hline Cellana sp. & 4.00 & --- & --- & --- & --- & --- & --- & --- & --- & --- & --- & --- \\
\hline Menathais turberosa & 4.00 & --- & 3.07 & --- & --- & --- & --- & --- & --- & --- & --- & --- \\
\hline Tylothais virgata & 9.33 & --- & --- & 7.14 & --- & --- & --- & --- & --- & 15 & --- & --- \\
\hline Menathais intermedia & --- & --- & --- & --- & 5.26 & --- & --- & --- & --- & --- & ---2 & 23.68 \\
\hline Indothais lacera & --- & --- & --- & --- & -- & --- & 2.85 & --- & 4.16 & --- & --- & --- \\
\hline Tenguella granulata & --- & --- & 12.3 & --- & --- & --- & --- & --- & --- & --- & --- & --- \\
\hline Vokesimurex tryoni & --- & --- & --- & --- & --- & --- & --- & 4.54 & --- & 2.50 & --- & --- \\
\hline Chicoreus brunneus & --- & --- & 1.53 & --- & --- & 11.11 & --- & --- & 4.16 & 2.50 & --- & --- \\
\hline Murex tribulus & 2.66 & --- & --- & --- & --- & --- & --- & --- & --- & --- & --- & --- \\
\hline Semiricinula konkanensis & 2.66 & --- & 3.07 & --- & --- & --- & --- & --- & --- & --- & --- & -- \\
\hline Thais sp. & --- & --- & --- & --- & --- & --- & --- & --- & --- & 2.50 & 33.33 & --- \\
\hline Morula nodicostata & --- & --- & --- & --- & --- & --- & --- & --- & 20.83 & --- & --- & --- \\
\hline Morula sp. & --- & --- & 1.53 & --- & --- & 8.33 & --- & --- & --- & --- & --- & --- \\
\hline Vasum turbinellus & 1.33 & --- & --- & --- & --- & --- & --- & 20.72 & --- & --- & --- & --- \\
\hline Clypeomorus bifasciata & 4.00 & --- & --- & --- & 5.26 & --- & 54.27 & 20.17 & --- & 5.00 & 33.33 & --- \\
\hline
\end{tabular}




\section{Continued}

\begin{tabular}{|c|c|c|c|c|c|c|c|c|c|c|c|c|}
\hline Clypeomorus petrosa & --- & --- & --- & --- & 5.26 & --- & - & 13.63 & --- & 2.50 & --- & --- \\
\hline Cerithium coralium & --- & --- & --- & --- & --- & --- & -- & --- & 4.16 & --- & --- & --- \\
\hline Rhinoclavis vertagus & --- & --- & --- & --- & --- & --- & -- & --- & --- & 2.50 & --- & --- \\
\hline Batillaria multformis & 8.00 & --- & 1.53 & --- & --- & --- & --- & --- & --- & --- & --- & --- \\
\hline Nebularia aurantia & --- & --- & 7.65 & --- & --- & --- & --- & --- & --- & --- & --- & --- \\
\hline Strigatella paupercula & --- & --- & 1.53 & --- & --- & --- & --- & --- & --- & --- & --- & --- \\
\hline Haliotis planata & --- & --- & --- & --- & --- & --- & --- & --- & --- & 2.50 & --- & --- \\
\hline Turritella $s p$ & --- & --- & --- & --- & --- & 2.77 & --- & --- & --- & --- & --- & --- \\
\hline Nassarius sp. & --- & --- & 3.07 & --- & --- & --- & --- & --- & --- & --- & --- & --- \\
\hline Nassarius coronatus & 1.33 & --- & --- & --- & 5.26 & --- & --- & --- & --- & --- & --- & --- \\
\hline Nassarius olivaceus & 1.33 & --- & --- & --- & --- & --- & --- & --- & --- & --- & --- & --- \\
\hline Nassarius pullus & 1.33 & --- & --- & --- & --- & --- & --- & --- & --- & --- & --- & --- \\
\hline Nassarius conoidalis & --- & --- & --- & --- & 5.26 & --- & --- & --- & --- & --- & --- & --- \\
\hline Oliva vidua & --- & --- & --- & --- & --- & 8.33 & --- & --- & --- & --- & --- & --- \\
\hline Oliva $s p$. & 2.66 & --- & --- & --- & --- & --- & --- & --- & --- & --- & --- & --- \\
\hline Bufonaria rana & --- & --- & 1.53 & --- & --- & --- & --- & --- & --- & --- & --- & --- \\
\hline Bufonaria echinata & 4.00 & --- & --- & --- & --- & --- & --- & --- & --- & --- & --- & --- \\
\hline Bursa granularis & 2.66 & --- & 1.53 & --- & --- & --- & --- & --- & --- & --- & --- & --- \\
\hline Gammula vagata & 1.33 & --- & --- & --- & --- & --- & -- & --- & --- & --- & --- & --- \\
\hline Turrilatirus craticulatus & --- & --- & --- & --- & --- & --- & --- & --- & 4.16 & --- & --- & --- \\
\hline Latirolagena smaragdulus & --- & --- & 1.53 & --- & --- & --- & --- & --- & --- & --- & --- & --- \\
\hline Scutellastra flexuosa & --- & --- & 1.53 & --- & --- & --- & --- & --- & --- & --- & --- & --- \\
\hline Terebralia paluster & --- & --- & --- & --- & --- & 2.77 & --- & --- & --- & --- & --- & --- \\
\hline Engina mendicaria & 1.33 & --- & --- & --- & --- & --- & --- & --- & 8.32 & --- & --- & --- \\
\hline Pollia undosa & 5.33 & --- & --- & --- & --- & --- & 2.85 & --- & 4.16 & --- & --- & --- \\
\hline Siphonaria sp.1 & --- & 5.88 & --- & --- & --- & --- & --- & --- & --- & --- & --- & --- \\
\hline Siphonaria sp.2 & --- & --- & 1.53 & --- & --- & 2.77 & --- & --- & --- & --- & --- & --- \\
\hline Canarium labiatum & 2.66 & 5.88 & --- & --- & --- & --- & --- & --- & --- & --- & --- & --- \\
\hline Gibberulus gibberulus & --- & --- & --- & 14.28 & --- & 2.77 & --- & --- & --- & --- & --- & --- \\
\hline Laevistrombus canarium & --- & --- & --- & 21.42 & --- & --- & --- & --- & --- & --- & --- & --- \\
\hline Conus ebraeus & --- & --- & --- & --- & --- & 2.77 & --- & --- & --- & --- & --- & --- \\
\hline Harpa major & 1.33 & --- & 1.53 & --- & --- & --- & --- & --- & --- & 5.00 & --- & 7.89 \\
\hline Semicassis bisulcata & 2.66 & --- & --- & --- & --- & --- & --- & --- & --- & --- & --- & --- \\
\hline Polinices mammilla & 1.33 & --- & --- & --- & 5,26 & --- & --- & --- & --- & --- & --- & --- \\
\hline Tanea lineata & --- & --- & --- & 7.14 & --- & 11.11 & --- & --- & --- & --- & --- & --- \\
\hline Patelloida saccharina & --- & --- & --- & - & --- & --- & --- & --- & --- & 15.00 & --- & --- \\
\hline Onchidium $s p$. & --- & --- & --- & 21.42 & --- & --- & --- & --- & --- & --- & --- & --- \\
\hline Littoraria undulate & --- & --- & --- & 21.42 & --- & 13.88 & --- & --- & --- & --- & --- & --- \\
\hline Monetaria moneta & --- & --- & --- & --- & --- & --- & --- & 9.08 & --- & --- & --- & --- \\
\hline Gyrineum natator & --- & --- & --- & --- & --- & 5.55 & --- & ---- & --- & --- & --- & \\
\hline
\end{tabular}


Further, the data were analysed by Primer 6 software [17] for the different diversity indices and cluster analysis. The cluster analysis was carried out based on the raw data which was converted for resemblance using Bray Courtis Similarity and then group average was taken as a cluster. The results were provided as a figurative form.

\section{Results}

Gastropoda species were collected from the coasts of Carbyns Cove, Rangat, Mayabunder and Diglipur for a period of one year in three prevailing seasons of Andaman and Nicobar Islands. They are SW monsoon, NE Monsoon and Non rainy Seasons. The collected samples were identified up to species level and their data were converted into percentages (Table 1). The photographs of these species were provided as Figures A1-A8.

Based on this study, among the four stations, seventy one species belonging to fifty two genus and thirty three families of gastropods were observed. Among the seventy one species, 38 species fell in unique category such that it may be noticed in any one of the stations, in any one of the season, i.e. their appearances/disappearance were not found to be site specific or seasonal specific (Table 2). Among them, 10 species available during the NE monsoon season in the station Rangat followed by SW monsoon season in the station Rangat (08), SW monsoon season in the station Mayabunder (05), NE monsoon season in the station Carbyns Cove (04), NE monsoon in the station Mayabunder, SW monsoon in the station Diglipur, represented 03 species each, Non rainy season of Mayabunder and Diglipur observed 01 species each, NE monsoon for Diglipur and Non rainy season in the station Rangat and Carbyns Cove noticed 01 species respectively, and the SW monsoon season in the station Carbyns Cove represented a total absence of gastropods.

Out of the remaining species, 20 species were observed in two occurrences by the way of any two seasons or any two locations or one season in two locations (Table 3). In these, NE monsoon in the station Rangat represented 09 species. Non Rainy season of Mayabunder (04) and SW monsoon in the station Rangat represented (07) species, followed by SW monsoon Mayabunder (05), Non Rainy season of Diglipur (02), NE monsoon of Diglipur (01), SW monsoon of Diglipur (03), NW monsoon of Carbyns Cove (02), SW monsoon of Carbyns Cove (02) and 1 species in Non rainy season of Rangat and Carbyns Cove were observed.

The species Tylothais virgata only observed during the NE monsoon of all the stations except Diglipur. Clypeomorus bifasciata species noticed only in the fifty percent of the seasons of the study period (NE Rangat, NRS Mayabunder, NE Diglipur, NRS Diglipur, NE and NRS of Carbyns Cove. Pollia undosa species were observed in NE monsoon season of Rangat and Diglipur as well as SW monsoon at Diglipur (Table 4).

Trochus radiates noticed in the NE monsoon of Rangat and Carbyns Cove 
Table 2. Percentage of One Time Occurrence of the Gastropoda Species in Different Locations and Seasons of the Present Studied Samples NER-NE Monsoon Rangat; NRRNon Rainy Season Rangat; SWR-SW Monsoon Rangat; NEM-NE Monsoon Mayabunder; NRM-Non Rainy Season Mayabunder; SWM-SW Monsoon Mayabunder; NED-NE Monsoon Diglipur; NRD-Non Rainy Season Diglipur; SWR-SW Monsoon Diglipur; NEC-NE Monsoon Carbyns Cove; NRC-Non Rainy Season Carbyns Cove; SWC- SW Monsoon Carbyns Cove.

\begin{tabular}{|c|c|c|c|c|c|c|c|c|c|c|c|c|c|}
\hline $\begin{array}{l}\text { Sl. } \\
\text { No. }\end{array}$ & Species Name & $\begin{array}{l}\text { NER } \\
2013\end{array}$ & $\begin{array}{l}\text { NRR } \\
2014\end{array}$ & $\begin{array}{l}\text { SWR } \\
2014\end{array}$ & $\begin{array}{l}2013 \\
2013\end{array}$ & $\begin{array}{l}\text { NRM } \\
2014\end{array}$ & $\begin{array}{l}\text { SWM } \\
2014\end{array}$ & $\begin{array}{l}\text { INED } \\
2013\end{array}$ & $\begin{array}{l}\text { NRD } \\
2014\end{array}$ & $\begin{array}{l}\text { SWD } \\
2014\end{array}$ & $\begin{array}{l}\text { NEC } \\
2013\end{array}$ & $\begin{array}{l}\text { NRC } \\
2014\end{array}$ & $\begin{array}{l}\text { SWC } \\
2014\end{array}$ \\
\hline 1 & Angaria delphinus & -- & -- & -- & 7.14 & -- & -- & -- & --- & -- & -- & -- & --- \\
\hline 2 & Lunella cinerea & 1.33 & --- & --- & --- & --- & --- & --- & --- & --- & --- & --- & --- \\
\hline 3 & Turbo sp. & --- & --- & --- & --- & --- & --- & --- & --- & --- & --- & 33.33 & --- \\
\hline 4 & Turbonilla felicita & --- & -- & --- & --- & --- & --- & --- & --- & --- & 2.50 & --- & --- \\
\hline 5 & Nerita histrio & --- & --- & --- & --- & --- & --- & 14.28 & -- & --- & --- & --- & --- \\
\hline 6 & Nerita insculpta & --- & --- & 15.38 & $3--$ & --- & --- & --- & --- & --- & --- & --- & --- \\
\hline 7 & Cellana radiate & 1.33 & --- & -- & --- & -- & --- & --- & --- & --- & -- & -- & --- \\
\hline 8 & Cellana sp. & 4.00 & --- & --- & --- & --- & --- & --- & --- & --- & --- & --- & --- \\
\hline 9 & Tenguella granulata & --- & --- & 12.30 & --- & --- & --- & --- & --- & --- & --- & --- & --- \\
\hline 10 & Murex tribulus & 2.66 & --- & --- & --- & --- & --- & --- & --- & --- & --- & --- & --- \\
\hline 11 & Morula nodicostata & --- & --- & --- & --- & --- & --- & --- & --- & 20.83 & -- & --- & --- \\
\hline 12 & Cerithium coralium & --- & --- & --- & --- & --- & --- & --- & --- & 4.16 & --- & --- & --- \\
\hline 13 & Rhinoclavis vertagus & --- & --- & --- & --- & --- & --- & --- & --- & --- & 2.50 & --- & --- \\
\hline 14 & Nebularia aurantia & --- & --- & 7.65 & --- & --- & --- & --- & --- & --- & --- & --- & --- \\
\hline 15 & Strigatella paupercula & --- & --- & 1.53 & --- & --- & --- & --- & --- & --- & --- & --- & --- \\
\hline 16 & Haliotis planata & --- & --- & --- & --- & --- & --- & --- & --- & --- & 2.50 & --- & --- \\
\hline 17 & Turritella $s p$ & --- & --- & --- & --- & --- & 2.77 & --- & --- & --- & --- & --- & --- \\
\hline 18 & Nassarius sp. & --- & --- & 3.07 & --- & --- & --- & --- & --- & --- & --- & --- & --- \\
\hline 19 & Nassarius olivaceus & 1.33 & --- & --- & --- & --- & --- & --- & --- & --- & --- & --- & --- \\
\hline 20 & Nassarius pullus & 1.33 & --- & --- & --- & --- & --- & --- & --- & --- & --- & --- & --- \\
\hline 21 & Nassarius conoidalis & --- & --- & --- & --- & 5.26 & --- & --- & --- & --- & --- & --- & --- \\
\hline 22 & Oliva vidua & --- & --- & --- & --- & --- & 8.33 & --- & --- & --- & --- & --- & --- \\
\hline 23 & Oliva sp. & 2.66 & --- & --- & --- & --- & --- & --- & --- & --- & --- & --- & --- \\
\hline 24 & Bufonaria rana & --- & --- & 1.53 & --- & -- & --- & --- & --- & --- & -- & -- & --- \\
\hline 25 & Bufonaria echinata & 4.00 & --- & --- & --- & --- & --- & --- & --- & --- & --- & --- & --- \\
\hline 26 & Gammula vagata & 1.33 & --- & --- & --- & --- & --- & --- & --- & --- & --- & --- & --- \\
\hline 27 & Turrilatirus craticulatus & --- & --- & --- & --- & --- & --- & --- & --- & 4.16 & --- & --- & --- \\
\hline 28 & Latirolagena smaragdulus & $s---$ & --- & 1.53 & --- & --- & --- & --- & --- & --- & -- & --- & --- \\
\hline 29 & Scutellastra flexuosa & --- & --- & 1.53 & --- & --- & --- & --- & --- & --- & --- & --- & --- \\
\hline 30 & Terebralia paluster & --- & --- & --- & --- & --- & 2.77 & --- & --- & --- & --- & --- & --- \\
\hline 31 & Siphonaria sp.1 & --- & 5.88 & -- & --- & --- & --- & --- & --- & --- & --- & -- & --- \\
\hline 32 & Laevistrombus canarium & --- & --- & -- & 21.42 & --- & --- & --- & --- & --- & --- & -- & --- \\
\hline 33 & Conus ebraeus & --- & --- & --- & --- & --- & 2.77 & --- & --- & --- & --- & --- & --- \\
\hline 34 & Semicassis bisulcata & 2.66 & --- & --- & --- & --- & --- & --- & --- & --- & --- & --- & --- \\
\hline 35 & Patelloida saccharina & --- & --- & --- & --- & --- & --- & --- & --- & --- & 15.00 & --- & --- \\
\hline 36 & Onchidium sp. & --- & --- & -- & 21.42 & -- & --- & --- & --- & --- & --- & -- & --- \\
\hline 37 & Monetaria moneta & --- & --- & --- & --- & --- & --- & --- & 9.08 & --- & --- & --- & --- \\
\hline 38 & Gyrineum natator & --- & --- & --- & --- & --- & 5.55 & --- & --- & --- & --- & --- & -- \\
\hline
\end{tabular}


Table 3. Percentage of Two Time Occurrence of the Gastropoda Species in Different Locations and Seasons of the Present Studied Samples NER-NE Monsoon Rangat; NRR-Non Rainy Season Rangat; SWR-SW Monsoon Rangat; NEM-NE Monsoon Mayabunder; NRM-Non Rainy Season Mayabunder; SWM-SW Monsoon Mayabunder; NEDNE Monsoon Diglipur; NRD-Non Rainy Season Diglipur; SWR-SW Monsoon Diglipur; NEC-NE Monsoon Carbyns Cove; NRC-Non Rainy Season Carbyns Cove; SWC-SW Monsoon Carbyns Cove.

\begin{tabular}{|c|c|c|c|c|c|c|c|c|c|c|c|c|c|}
\hline $\begin{array}{l}\text { Sl. } \\
\text { No. }\end{array}$ & Species Name & $\begin{array}{l}\text { NER } \\
2013 \\
\end{array}$ & $\begin{array}{l}\text { NRR } \\
2014\end{array}$ & $\begin{array}{l}\text { SWR } \\
2014\end{array}$ & $\begin{array}{l}\text { NEM } \\
2013\end{array}$ & $\begin{array}{l}\text { NRM } \\
2014\end{array}$ & $\begin{array}{l}\text { SWM } \\
2014\end{array}$ & $\begin{array}{l}\text { NED } \\
2013\end{array}$ & $\begin{array}{l}\text { NRD } \\
2014\end{array}$ & $\begin{array}{l}\text { SWD I } \\
20142\end{array}$ & $\begin{array}{l}\text { NEC } \\
2013\end{array}$ & $\begin{array}{l}\text { NRC } \\
2014\end{array}$ & $\begin{array}{l}\text { SWC } \\
2014\end{array}$ \\
\hline 1 & Tectus pyramis & ---- & --- & --- & --- & 10.52 & --- & --- & --- & 4.16 & --- & --- & --- \\
\hline 2 & Nerita costata & --- & --- & 13.84 & -- & --- & --- & --- & --- & --- & --- & --- & 15.78 \\
\hline 3 & Menathais turberosa & 4.00 & --- & 3.07 & --- & --- & --- & --- & --- & --- & --- & --- & --- \\
\hline 4 & Menathais intermedia & --- & --- & --- & --- & 5.26 & --- & --- & --- & --- & --- & --- & 23.68 \\
\hline 5 & Indothais lacera & --- & --- & --- & --- & --- & --- & 2.85 & --- & 4.16 & --- & --- & --- \\
\hline 6 & Vokesimurex tryoni & --- & --- & --- & --- & --- & --- & --- & 4.54 & --- & 2.5 & --- & --- \\
\hline 7 & Semiricinula konkanensis & 2.66 & --- & 3.07 & --- & --- & --- & --- & --- & --- & --- & --- & -- \\
\hline 8 & Thais sp. & --- & --- & --- & --- & --- & --- & --- & --- & --- & 2.5 & 33.33 & --- \\
\hline 9 & Morula sp. & --- & --- & 1.53 & --- & --- & 8.33 & --- & --- & --- & --- & --- & --- \\
\hline 10 & Vasum turbinellus & 1.33 & --- & --- & --- & --- & --- & --- & 20.72 & --- & --- & --- & --- \\
\hline 11 & Batillaria multformis & 8.00 & --- & 1.53 & --- & --- & --- & --- & --- & --- & --- & --- & --- \\
\hline 12 & Bursa granularis & 2.66 & --- & 1.53 & --- & --- & --- & --- & --- & --- & --- & --- & --- \\
\hline 13 & Engina mendicaria & 1.33 & --- & --- & --- & --- & --- & --- & --- & 8.32 & --- & --- & --- \\
\hline 14 & Siphonaria sp.2 & --- & --- & 1.53 & --- & --- & 2.77 & -- & --- & --- & --- & --- & --- \\
\hline 15 & Canarium labiatum & 2.66 & 5.88 & --- & --- & --- & --- & --- & --- & --- & --- & --- & --- \\
\hline 16 & Gibberulus gibberulus & --- & --- & --- & 14.28 & --- & 2.77 & --- & --- & --- & --- & --- & --- \\
\hline 17 & Polinices mammilla & 1.33 & --- & --- & --- & 5.26 & --- & --- & --- & --- & --- & --- & --- \\
\hline 18 & Tanea lineata & --- & --- & --- & 7.14 & --- & 11.11 & --- & --- & --- & --- & --- & --- \\
\hline 18 & Littoraria undulate & --- & --- & --- & 21.42 & --- & 13.88 & --- & --- & --- & --- & --- & --- \\
\hline 20 & Nassarius coronatus & 1.33 & --- & --- & --- & 5.26 & --- & --- & --- & --- & --- & --- & --- \\
\hline
\end{tabular}

and SW monsoon period in Mayabunder and Rangat. Chicoreus brunneus observed in SWM of Rangat, Mayabunder and Diglipur as well as NEM of Carbyns Cove stations. SWM of Mayabunder, NEM of Rangat and Carbyns exhibited Monodonta australis species and in Diglipur it had been noticed in Non rainy season and SW monsoon. Harpa major species were noticed in NEM and SWM of Rangat and Carbyns Cove (Table 4).

Except NEM in Mayabunder and NRS of Carbyns Cove all the remaining seasons represented by Nerita albicilla (Table 4). This is the only species observed in almost all the stations with the majority of the seasons (Table 4).

The NEM in Rangat, Diglipur and Carbyns Cove as well as NRS in Diglipur and SWM in Mayabunder and Diglipur represented the species Turbo bruneus, i.e. all the three seasons in Diglipur this species was present. Nerita oryzarum was observed during the period of NEM of Rangat, Diglipur and Carbyns Cove, 
Table 4. Percentage of Special Occurrence of the Gastropoda Species in Different Locations and Seasons of the Present Studied Samples NER-NE Monsoon Rangat; NRR-Non Rainy Season Rangat; SWR-SW Monsoon Rangat; NEM-NE Monsoon Mayabunder; NRM-Non Rainy Season Mayabunder; SWM-SW Monsoon Mayabunder; NED-NE Monsoon Diglipur; NRD-Non Rainy Season Diglipur; SWR-SW Monsoon Diglipur; NEC-NE Monsoon Carbyns Cove; NRC-Non Rainy Season Carbyns Cove; SWC-SW Monsoon Carbyns Cove.

\begin{tabular}{|c|c|c|c|c|c|c|c|c|c|c|c|c|c|}
\hline $\begin{array}{r}\text { Sl. } \\
\text { No. }\end{array}$ & Species Name & $\begin{array}{l}\text { NER } \\
2013\end{array}$ & $\begin{array}{l}\text { NRK } \\
2014\end{array}$ & 2014 & 2013 & 2014 & $\begin{array}{l}\text { SWM } \\
2014\end{array}$ & $\begin{array}{l}\text { NED } \\
2013\end{array}$ & $\begin{array}{l}\text { NRD } \\
2014 \\
\end{array}$ & $\begin{array}{l}\text { SWD } \\
2014\end{array}$ & $\begin{array}{l}\text { NEC } \\
2013\end{array}$ & $\begin{array}{l}\text { NRC } \\
2014\end{array}$ & $\begin{array}{l}\text { SWC } \\
2014\end{array}$ \\
\hline 1 & Trochus radiates & 5.32 & --- & 1.53 & --- & --- & 2.77 & --- & --- & --- & 5.00 & --- & --- \\
\hline 2 & Monodo & 1.33 & --- & --- & --- & .. & 8.33 & -- & 4.54 & 4.16 & 7.50 & --- & --- \\
\hline 3 & Wlohinus & & --- & --- & 7.14 & --- & & --- & --- & --- & --- & --- & --- \\
\hline 4 & Trita albicill & 5.33 & 88.23 & 10.76 & --- & 5.26 & 8.33 & 17.1 & 13.63 & 20.83 & 25.00 & --- & 52.62 \\
\hline 5 & Terita & 1.33 & --- & 7.68 & --- & 36.84 & -- & 2.85 & --- & 8.32 & 2.50 & --- & --- \\
\hline 6 & Tyloth & 9.33 & --- & --- & 7.14 & -- & --- & --- & --- & --- & 1.50 & --- & --- \\
\hline 7 & Chico & - & --- & 1.53 & --- & --- & 11.11 & --- & --- & 4.16 & 2.50 & --- & --- \\
\hline 8 & Clypeo & 4.00 & --- & --- & --- & 5.26 & --- & 54.27 & 20.17 & --- & 5.00 & 33.33 & --- \\
\hline 9 & Pollia 2 & 5.33 & --- & --- & --- & -- & --- & 2.85 & --- & 4.16 & --- & --- & --- \\
\hline 10 & Harpa major & 1.33 & --. & 1.53 & --- & -.- & --. & --- & -.- & --- & 5.00 & --- & 7.89 \\
\hline
\end{tabular}

SWM in Rangat and Diglipur and NRS in Mayabunder (Table 4).

The station Carbyns Cove exhibited a total of nineteen species consist of nine families. Out of nineteen species, single occurrence was noticed in 15 species and dual occurrence noticed for 4 species. A total of 24 families was missing in this station.

The station Rangat reported 42 species from 21 families. Out of 42 species single seasons were noticed for 32 species and dual occurrence noticed in 09 species and one species in all seasons. Twelve families were missing from this station.

The gastropods of 29 species belonging to 17 families were observed at Mayabunder station. Out of these 29 species, single occurrence noticed in 25 species and dual occurrence noticed in four species. There were 16 families missing.

Diglipur station reported 20 species belonging to 10 families in all the seasons. Out of 20 species, 13 species single time reported and two and three time presence noticed respectively 05 and 02 species. In this location 13 families were missing.

The study period represented the highest number of species (60) during the NE monsoon represented than remaining seasons. Next higher number of species (53) noticed in the SW monsoon and lowest numbers noticed in Non rainy season (26).

The results of the studied gastropods in the Primer software and the univariate analysis (Table 5) suggested that highest Margalefs Species Richness Index (6.080) noticed during the NE monsoon period of Rangat (29 species) and the lowest (0.434) noticed during Non Rainy Season at Carbyns Cove and Rangat (3 
species). However, the Pielou's Evenness Index represented the maximum of 1.000 for the Carbyns Cove Non Rainy Season samples (3 species) and lowest (0.404) noticed for the Rangat (3 species) Non Rainy Season. The ShannonWiener Index represented the maximum (3.067) for the NE monsoon period of Rangat (29 species) and the lowest (0.444) noticed for the Rangat (3 species) Non Rainy Season. The Simpson Diversity Index supports the Shannon-Weiner Index.

The group average of the Cluster plot (Figure 2) suggested that three major clusters divided in one independent character and two with sub clusters. The one independent cluster represented the Non Rainy Season of Carbyns Cove (Cluster A). The second cluster with two sub clusters represented NE and SW monsoon periods of Mayabunder (Cluster B-B1, B2). The third cluster (Cluster C) with nine sub clusters has represented by five units of the remaining seasons and stations. The first unit (C-1) has two sub-sub-clusters (C-1-1 and 2) for NRS Rangat and SWM Carbyns Cove. The unit two (C-2) has two sub-sub-clusters represented NEM Digilipur and NRS Diglipur.

The unit 3 (C-3) also has two sub-sub clusters with SWM Digilipur and NEM Carbyns Cove. The unit 4 (C-4) represented only one season that is NRS Mayabunder. The last unit 5 (C-5) has two subdivisions represented NEM and SWM Rangat.

Table 5. Primer 6 Software Univariate Analytical Result. NER-NE Monsoon Rangat; NRR-Non Rainy Season Rangat; SWR-SW Monsoon Rangat; NEM-NE Monsoon Mayabunder; NRM-Non Rainy Season Mayabunder; SWM-SW Monsoon Mayabunder; NED-NE Monsoon Diglipur; NRD-Non Rainy Season Diglipur; SWR-SW Monsoon Diglipur; NEC-NE Monsoon Carbyns Cove; NRC-Non Rainy Season Carbyns Cove; SWCSW Monsoon Carbyns Cove.

\begin{tabular}{|c|c|c|c|c|c|c|}
\hline Season & $\begin{array}{c}\text { Total } \\
\text { Species } \\
(\mathrm{S})\end{array}$ & $\begin{array}{c}\text { Total } \\
\text { Individuals } \\
(\mathrm{N})- \\
\text { Normalised }\end{array}$ & $\begin{array}{l}\text { Margalef's } \\
\text { Species } \\
\text { Richness } \\
\text { Index (d) }\end{array}$ & $\begin{array}{l}\text { Pielou's } \\
\text { Evenness } \\
\text { Index } \\
\left(J^{\prime}\right)\end{array}$ & $\begin{array}{c}\text { Shannon } \\
\text { Wiener } \\
\text { Index (H') }\end{array}$ & $\begin{array}{c}\text { Simpson } \\
\text { Diversity } \\
\text { Index } \\
(1-\lambda)\end{array}$ \\
\hline NER & 29 & 100 & 6.0801 & 0.9109 & 3.0673 & 0.9466 \\
\hline NRR & 3 & 100 & 0.4343 & 0.4038 & 0.4437 & 0.2166 \\
\hline SWR & 21 & 100 & 4.3429 & 0.8711 & 2.6521 & 0.9184 \\
\hline NEM & 7 & 100 & 1.3028 & 0.9424 & 1.8338 & 0.8349 \\
\hline NRM & 10 & 100 & 1.9543 & 0.8596 & 1.9794 & 0.8105 \\
\hline SWM & 16 & 100 & 3.2572 & 0.9407 & 2.6082 & 0.9259 \\
\hline NED & 8 & 100 & 1.5200 & 0.6825 & 1.4192 & 0.6578 \\
\hline NRD & 9 & 100 & 1.7372 & 0.9330 & 2.0499 & 0.8650 \\
\hline SWD & 12 & 100 & 2.3886 & 0.9071 & 2.2542 & 0.8802 \\
\hline NEC & 16 & 100 & 3.2572 & 0.8618 & 2.3893 & 0.8826 \\
\hline NRC & 3 & 100 & 0.4343 & 1 & 1.0986 & 0.6734 \\
\hline SWC & 4 & 100 & 0.6514 & 0.8445 & 1.1708 & 0.6421 \\
\hline
\end{tabular}




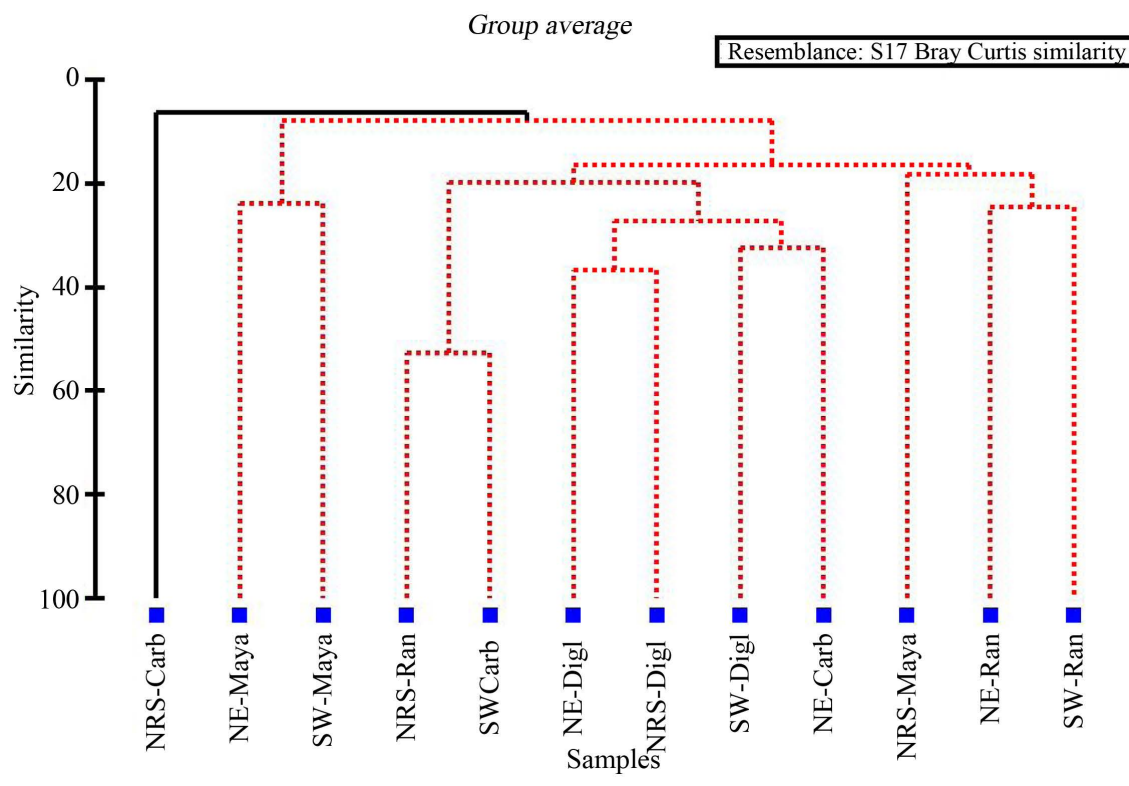

Figure 2. Cluster dendrogram for the studied gastropoda distribution.

\section{Discussion}

Based on the above results it was inferred that the station Rangat was highly enriched with gastropod species when comparing all the four stations followed by Mayabunder, Diglipur and Carbyns Cove stations. However, based on the occurrence of the species, it was noted that the stations Rangat and Carbyns Cove may be similar in environmental condition than the remaining two stations. Similarly, NE and SW monsoon plays a major role in the diversity and distribution of species in Rangat stations than in the Non rainy season. Mayabunder and Diglipur stations exhibited less diversity and distribution in NE monsoon than SW monsoon.

However, the Non Rainy Season shows diversity and distribution in between the above rainy seasons. Carbyns Cove represented SWM and NRS less favorable for diversity and distribution than NEM period.

Out of seventy one species studied, 10 species identified only upto genus level. The remaining 61 species, 57 species already reported in Andaman and Nicobar Islands by Rao (2003). The species Nerita oryzarum was reported by Ragunathan and Venkatraman [18] and the other three species Turbonilla felicita, Vokesimurex tryoni and Batillaria multiformis were not reported in these Islands.

The west coast of India in Raigad District, Maharashtra exhibited 14 species occurrence from the studies of Kade and Mane [19]. Haragi et al. [20] reported 7 species at the Karwar coast of Karnataka. Seven species of Charwad coast (Gujarat) reported by Vadher et al. [21]. Goa coast exhibited seven species of occurrence [6]. Elaiyaraja et al. [22] reported four similar species in the study from Palk Bay region. Chelladurai et al. [23], reported one studied species from the Gulf of Mannar, Tamil Nadu region. Sakthivel and Fernando [24] studied five species in the present study from Mudasal Odai and Nagapatinam coastal regions. 
Arumaugam et al. [8] reported eighteen species of the present study in the Great Nicobar Coastal environment. Priyankadevi et al. [25] reported 12 species of the present study in Avis Island and Karmatang beach of Mayabunder.

The station Rangat, Mayabunder, Diglipur and Carbyns Cove represented 42, 29, 20 and 19 species among the studied species. This suggested that Rangat station has an exposure of rocky outcrop which may provide a better niche for larger diversity of gastropod species. Even though Carbyns Cove also has the similar exposure because of little areal exposure during the low tide time may be reduced the population of this species. The studied Mayabunder location may have rocky and mangrove exposure support the presence of Littoraria undulata exclusively in this location and have next in concentration of diversity. Diglipur station was covered with coralline exposure than the rock exposure has fallen in between the Carbyns Cove and Mayabunder diversity status. Chelladurai et al. [23], and Elaiyaraja et al. [22], reported that the coralline outcrop has more amount of diversity during the non rainy season also proved in this study location where the similar outcrop exposed, i.e. the station Diglipur which exhibited comparatively equal or more diversity during the non rainy season than in the monsoon period.

These studies well follow the existing inference from the world wide study pattern of the gastropoda. As reported by the Sanpanich et al. [26] and Suresh et al. [27], Littoraria undulata only occurred in the rocky outcrop with mangrove environment. Ramadoss [28], Tan and Clements [29], Arumugam et al. [8], Haragi et al. [20], Khade and Mane [19], Sorensen [30], Miloslavich et al. [31] and David [6] was reported rocky outcrop species were also present in this study area also in the same environment. Bhadja [32], David [6] and Vadher et al. [21], reported that diversity is more in the period of monsoon than in non rainy seasons of rocky outcrop environment also supported in this present study that the station Rangat exposed to 40 species during the monsoon (SW and NE) period. Yaninek [33] reported that Conus ebraeus, more abundant in the higher intertidal zone than in the outer zone, which also true in the present study.

The results of univariate analysis based on Margalef's Species Richness Index, Pielou's Evenness Index, Shannon-Wiener Index and Simpson Diversity Index supported each other for the distribution of gastropods in the study locations for all the seasons. The cluster analysis also grouped the unique character of occurrences with reference to locations and seasons. While comparing these clusters, it was noted that that the individual clusters Cluster A and Cluster B has no similar species between them, but it shared similarities of the species with $\mathrm{C} 1$ and C2 clusters. The cluster B represented single common species (Nerita albicilla). Cluster C, among all the sub clusters (5 Nos.), the cluster four and five have three common species Nerita albicilla, Nerita oryzarum and Nerita polita. The sub cluster three, four and five showing two common species Nerita albicilla and Nerita oryzaraum and three and four exhibited two common species Nerita albicilla and Turbo brunneus. However, the cluster two and five exhibited one 
common species are Nerita albicilla. Turbo species exclusively in Cluster A among the three available species and Cluster B exhibited twelve species exclusively among the 20 available species. Total forty nine species exclusively found in Cluster $\mathrm{C}$ among 59 which can be divided into five sub clusters.

\section{Conclusion}

The present study of gastropods distribution in the South, Middle and North Andaman groups of Islands suggested that there are 71 species belonging to 52 genus and 33 families. At any one of the time and any one of the locations, only one occurrence was noticed for 38 species and remaining 33 species were overlapping with respect to stations and seasons. Nerita albicilla observed at all the stations with the majority of the seasons except NEM of Mayabunder and NRS of Carbyns Cove. Turbo species exclusively in Cluster A among the three available species and Cluster B exhibited twelve species exclusively among the 20 available species. Total forty nine species exclusively found in Cluster $\mathrm{C}$ among 59 which can be divided into five sub clusters. This study also concluded that three species (Turbonilla felicita, Vokesimurex tryoni and Batillaria multiformis) were new to the Andaman Islands and among them two species (Vokesimurex tryoni and Batillaria multiformis) were not recorded any part of India.

\section{Acknowledgements}

The authors thank the Central Marine Living Resources and Ecology, Ministry of Earth Sciences, Kochi for funding this work (MOES/CMLRE/10-ITIS/5/2012, Date: 01/10/2012). They also acknowledge the authorities of Pondicherry University for providing the facilities needed for the execution of this project.

\section{References}

[1] Venkataraman, K. and Wafer, M. (2005) Coastal and Marine Biodiversity of India. Indian Journal of Marine Sciences, 34, 57-75.

[2] Rao, S.N.V. and Dey, A. (2000) Catalogue of Marine Molluscs of Andaman and Nicobar Islands. Records of Zoological Survey of India, Zoological Survey of India, Kolkatta, India. Occ. Paper No. 187, 1-323.

[3] Benkendorff, K. and Davis, A.R. (2002) Identifying Hotspots of Molluscan Species Richness on Rocky Intertidal Reefs. Biodiversity and Conservation, 11, 1959-1973. https://doi.org/10.1023/A:1020886526259

[4] Chiba, S. (2007) Species Richness Patterns along Environmental Gradients in Island Land Molluscan Fauna Ecology. Ecological Society of America, 88, 1738-1746.

[5] Benkendorff, K. and Przeslawski, R. (2008) Multiple Measures Are Necessary to Assess Rarity in Macro-Molluscs: A Case Study from Southeastern Australia. Biodiversity and Conservation, 17, 2455-2478. https://doi.org/10.1007/s10531-008-9392-6

[6] David, A. (2013) Biodiversity and Distribution of Marine Gastropods (Mollusca) during Pre- and Post-Monsoon Seasons along the Goa Coastline, India. Journal of Marine Biological Association of India, 55, 17-24. https://doi.org/10.6024/jmbai.2013.55.1.01720-03 
[7] Shanmugam, A. and Vairamani, S. (2015) Molluscs in Mangroves: A Case Study, Centre of Advanced Study in Marine Biology. Annamalai University, 371-382.

[8] Arumugam, M., Shanmugam, A., Balasubramanian, T., Kannan, I. and Ajmalkhan, S. (2010) Studies on Molluscan Diversity of Great Nicobar Island: A Pre Tsunami Scenario. In: Sivaperuman, C., Ra-Gunathan, C. and Venketraman, R., Eds., Recent Trends in Biodiversity of Andaman and Nicobar Islands, Zoological Survey of India, Kolkatta, 275-282.

[9] Pinn, F. (1990) Sea Snails of Pondicherry. Nehru Science Center, Pondicherry, 215 p.

[10] Apte, D. (1998) The Book of Indian Shells. Oxford University Press, Mumbai, 1-187.

[11] Apte, D. (2002) Field Guide to the Marine Life of India. Stusa Mudra Pvt. Ltd., India, 160-223.

[12] Jereb, P. and Rober, C.F.E. (2005) Cephalopods of the World. An Annotated and Illustrated Catalogue of Cephalopod Species Known to Date. Volume 1. Chambered Nautiluses and Sepioids (Nautilidae, Sepiidae, Sepiolidae, Sepiadariidae, Idiosepiidae and Spirulidae). FAO Species Catalogue for Fishery Purpose, No. 4, Vol. 1. FAO, Rome, 1-262.

[13] Ramakrishna and Dey, A. (2003) Manual on the Identification of Schedule Molluscs from India. Zoological Survey of India, Kolkata, $40 \mathrm{p}$.

[14] Rao, S.N.V. (2003) Indian Sea Shells (Part-1) Polyplaeophora and Gastropoda. Records of Zoological Survey of India, Occ. Paper No. 192. Zoological Survey of India. Kolkata, India, I-X, 1-416.

[15] D'souza, N.C. (2005) A Survey of Marine Molluscan Shells in Andaman and Nicobar Islands. Master's Thesis, Pondicherry University, Port Blair, 58 p.

[16] WoRMS Editorial Board (2018) World Register of Marine Species. http://www.marinespecies.org

[17] Primer-6. (2006) Plymouth Routine in Multivariate Ecological Research Software. Version 6. In: Clarke, K.R. and Gorley, G.N., Eds., Plymouth Marine Laboratory, Plymouth, 1-190.

[18] Ragunathan, C. and Venkatraman, K. (2012) Diversity and Distribution of Coral and Their Associated Fauna of Rani Jhansi Marine National Park, Andaman and Nicobar Islands. In: Venkatraman, K., Ragunathan, C. and Sivaperuman, C., Eds., Ecology of Faunal Communities on the Andaman and Nicobar Islands, Zoological Survey of India, Kolkatta, 177-208. https://doi.org/10.1007/978-3-642-28335-2_12

[19] Khade, S.N. and Mane, U.H. (2012) Diversity of Bivalve and Gastropod, Molluscs of Some Localities from Raigad District, Maharashtra, West Coast of India. Recent Research in Science and Technology, 4, 43-48.

[20] Haragi, S.B., Ulhas, G. and Naik, U.G. (2012) Resilient Intertidal Biodiversity Profile of Majali Coast, Karwar, West Coast of India. CIBTech Journal of Zoology, 1, 68-78.

[21] Vadher, P., Gadhvi, I.R., Parekh, H. and Dabhi, J. (2014) Occurrence of Marine Molluscan along the Chorwad Coast, Gujarat-India. Advances in Applied Science Research, 5, 24-28.

[22] Elaiyaraja, C., Rajasekaran, R. and Sekar, V. (2013) Check List and Occurrence of Marine Gastropoda long the Palk Bay Region, Southeast Coast of India. Pelagia Research Library Advances in Applied Science Research, 4, 195-199.

[23] Chelladurai, G., Mohanraj, J. and Sasirakhamani, M. (2013) Distribution of Marine Gastropods in Tuticorin Coast, Gulf of Mannar, India. International Journal of Pharmaceutical and Biological Archives, 4, 371-374. 
[24] Sakthivel, K. and Fernando, S.A. (2014) Gastropod Diversity in Mudasal Odai and Nagapattinam, Southeast Coast of India. Indian Journal of Geo-Marine Sciences, 43, 519-527.

[25] Priyankadevi, Revathi, K. and Raghunathan, C. (2015) A Study on Diversity of Molluscs in Maya-Bunder Islands. Global Journal for Research Analysis, 4, 10.

[26] Sanpanich, K., Wells, F.E. and Chitramvong, Y. (2004) Distribution of the Family Littorinidae (Mollusca: Gastropoda) in Thailand. Records of the Western Australian Museum, 22, 241-251. https://doi.org/10.18195/issn.0312-3162.22(3).2004.241-251

[27] Suresh, M., Arularasan, S. and Ponnusamy, K. (2012) Distribution of Molluscan Fauna in the Artificial Mangroves of Pazhayar Back Water Canal, Southeast Coast of India. Advances in Applied Science Research, 3, 1795-1798.

[28] Ramadoss, K. (2003) Status of Exploited Marine Fishery Resources of India. In: Joseph, M.M. and Jayaprakash, A.A., Eds., Central Marine Fisheries Research Institute, Kochi, 201-210.

[29] Tan, S.K. and Clements, R. (2008) Taxonomy and Distribution of the Neritidae (Mollusca: Gastropoda) in Singapore. Zoological Studies, 47, 481-494.

[30] Sørensen, A.M. (2012) Distribution, Diversity and Abundance Patterns of Intertidal Benthic Molluscs on Rocky Shores (Phuket Island, Thailand). The Thailand Natural History Museum Journal, 6, 113-128.

[31] Miloslavich, P., Cruz-Motta, J.J., Klein, E., Iken, K., Weinb, V., et al. (2013) Large-Scale Spatial Distribution Patterns of Gastropod assemblages in Rocky Shores. PLoS ONE, 8, e71396. https://doi.org/10.1371/journal.pone.0071396

[32] Bhadja, P. (2010) Marine Wealth of Saurashtra Coast: Spatial and Temporal Variations in the Seawater Quality and Its Role in Intertidal Assemblage and Macrofaunal Diversity around Anthropogenically Influenced Shore. Unpublished Ph.D. Thesis, Saurashtra University, Rajkot, 1-210.

[33] Yaninek, J.S. (1978) A Comparative Survey of Reef-Associated Gastropods at Maziwi Island, Tanzania. Journal of the East Africa Natural History Society and National Museum, 31, 1-16. 


\section{Appendix}

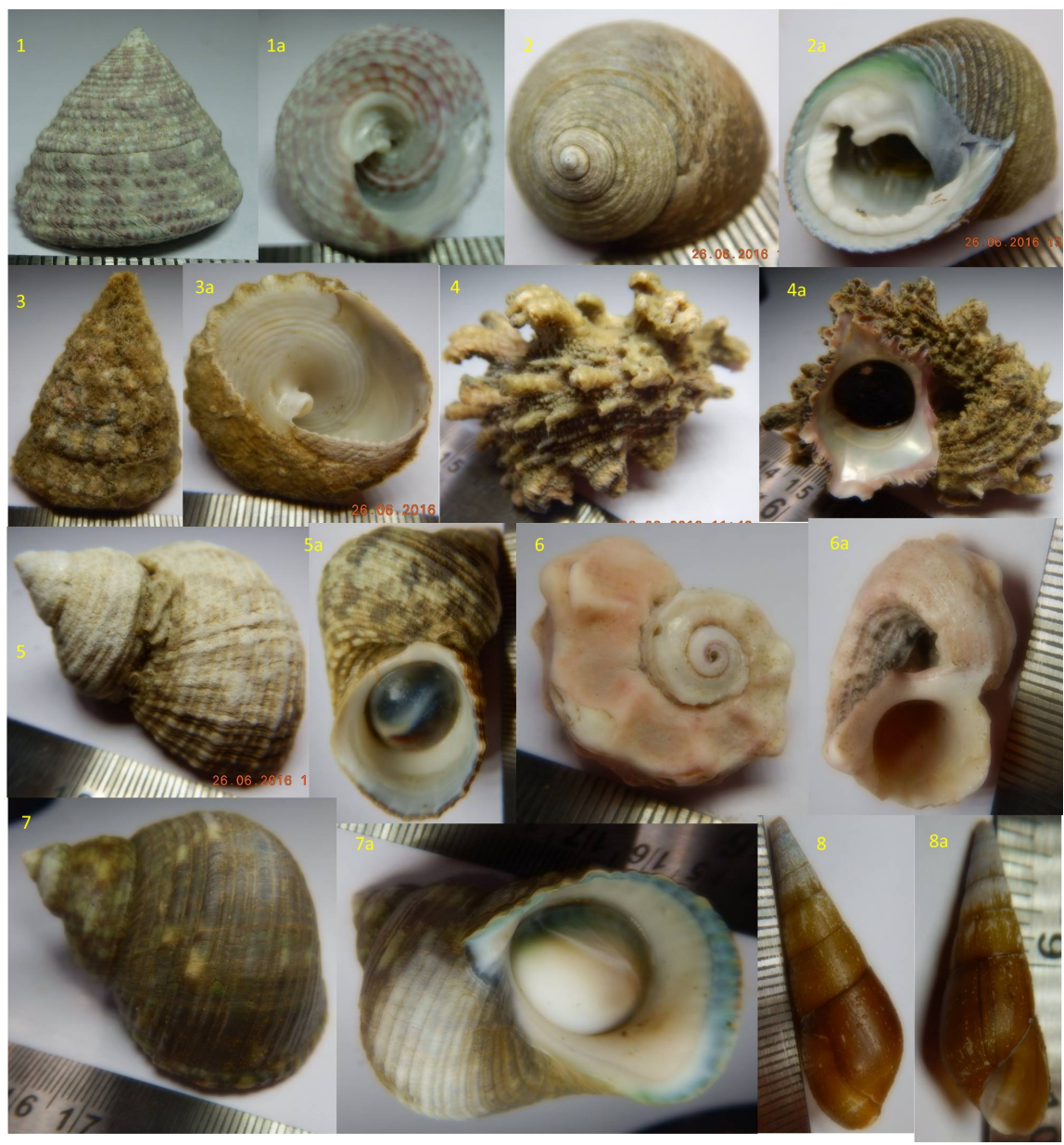

Figure A1. 1. Trochus radiatus (lateral view); 1a. Trochus radiatus (apertural view); 2. Monodonta austalis (lateral view); 2a. Monodonta australis (apertural view); 3. Tectus pyramis (lateral view); 3a. Tectus pyramis (apertural view); 4. Angaria delphinus (lateral view); 4a. Angaria delphinus (apertural view); 5. Turbo brunneus (lateral view); 5a. Turbo brunneus (apertural view); 6. Lunella cinerea (lateral view); 6a. Lunella cinerea (apertural view); 7. Turbo sp. (lateral view); 7a. Turbo sp. (apertural view); 8. Turbonilla felicita (lateral view); 8a. Turbonilla felicita (apertural view). 


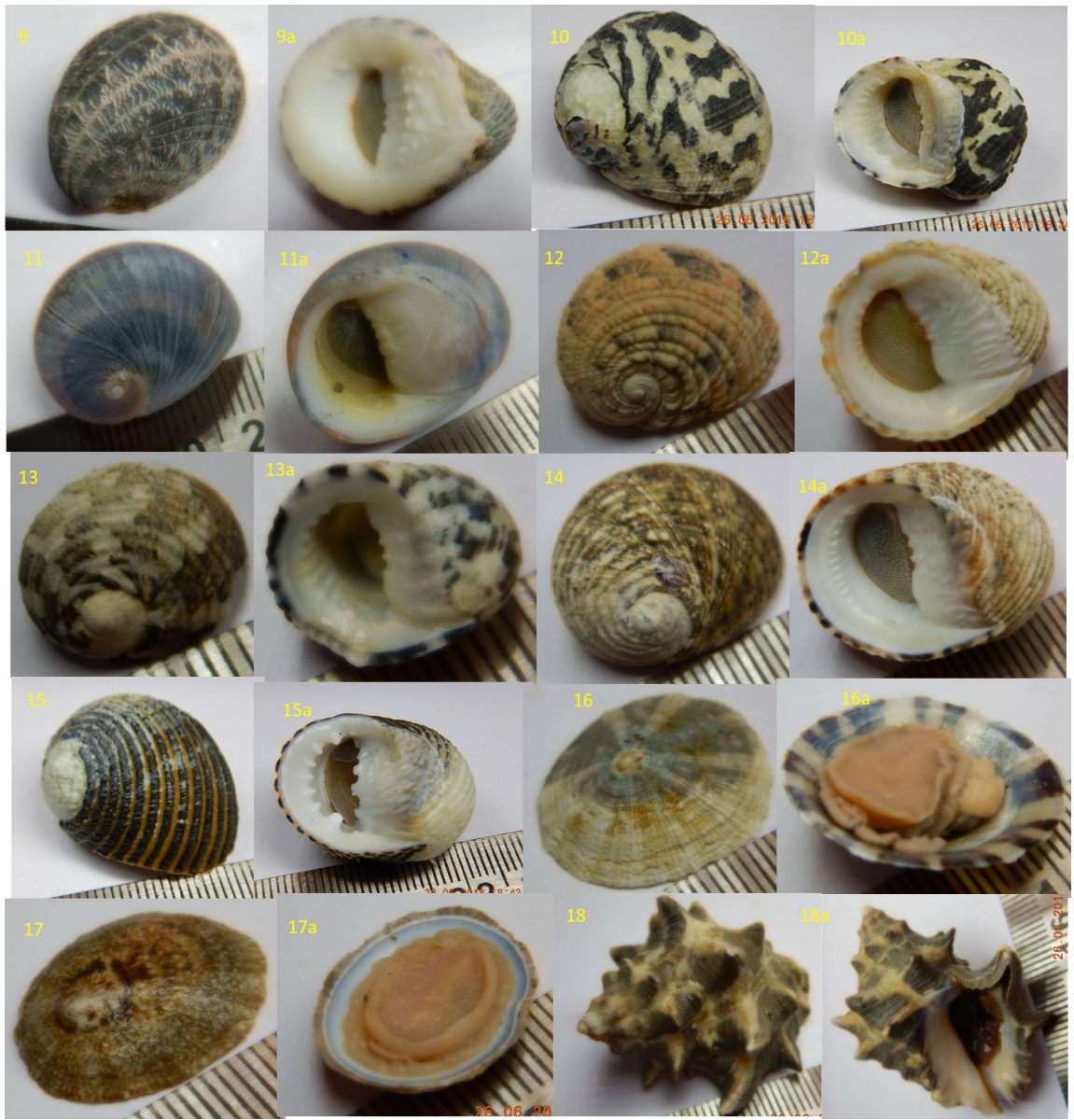

Figure A2. 9. Nerita albicilla (lateral view); 9a. Nerita albicilla (apertural view); 10. Nerita oryzarum (lateral view); 10a.Nerita oryzarum (apertural view); 11. Nerita polita (lateral view); 11a. Nerita polita (apertural view). 12. Nerita histrio (lateral view); 12a. Nerita histrio (apertural view); 13. Nerita chamaeleon (lateral view); 13a. Nerita chamaeleon (apertural view); 14. Nerita insculpta (lateral view); 14a. Nerita insculpta (apertural view); 15. Neita costata (lateral view); 15a. Nerita costata (apertural view); 16. Cellana radiata (lateral view); 16a. Cellana radiata (apertural view); 17. Cellana sp. (lateral view); 17a. Cellana sp. (apertural view); 18. Menathais tuberosa (lateral view); 18a. Menathais tuberosa (apertural view). 


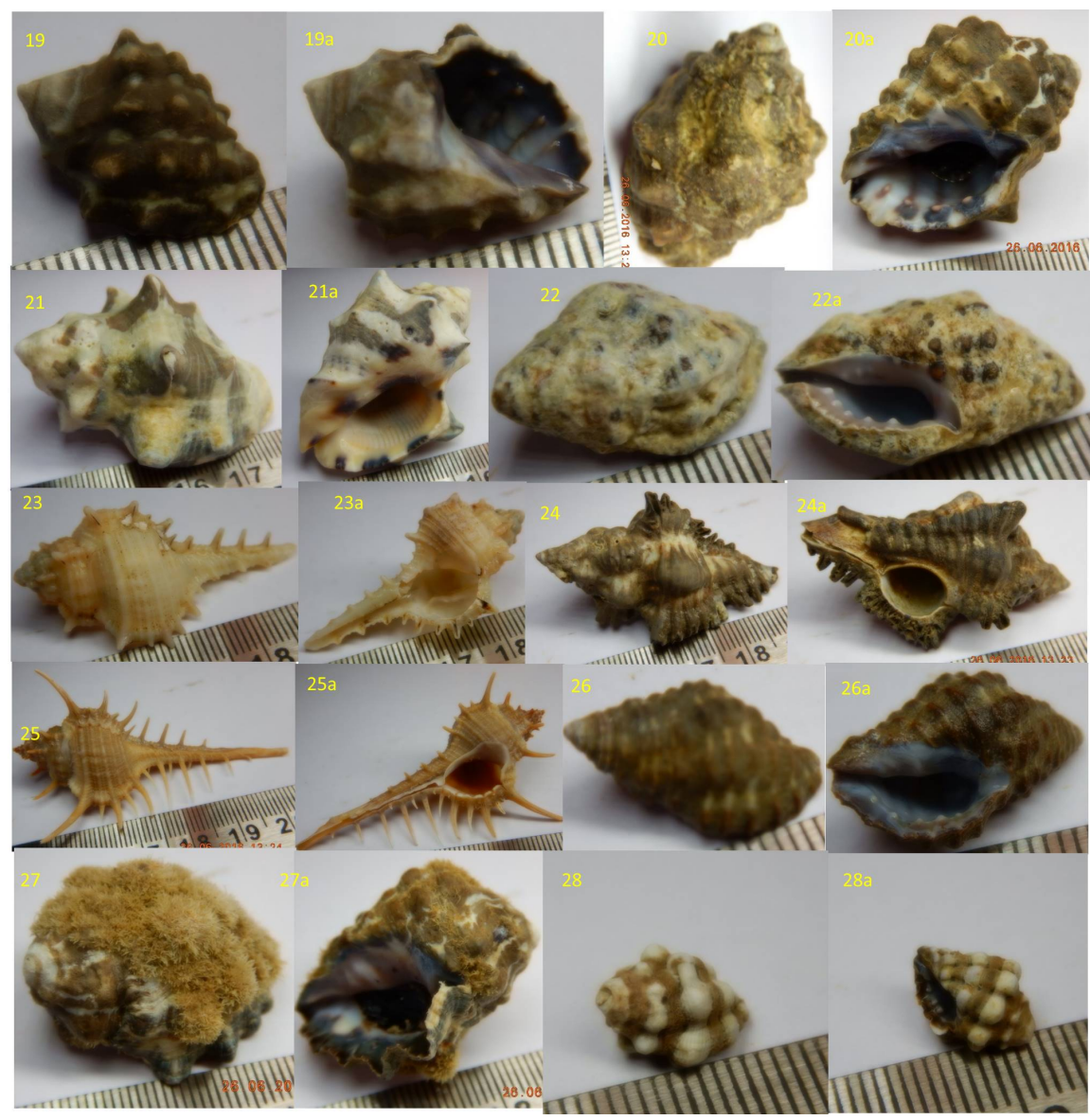

Figure A3. 19. Tylothais virgata (lateral view); 19a. Tylothais virgata (apertural view); 20. Menathais intermedia (lateral view); 20a. Menathais intermedia (apertural view). 21. Indothais lacera (lateral view); 21a. Indothais lacera (apertural view); 22. Tenguella granulata (lateral view); 22a. Tenguella granulata (aperture); 23. Vokesimurex tryoni (lateral view); 23a. Vokesimurex tryoni (apertural view); 24. Chicoreus brunneus (lateral view); 24a. Chicoreus brunneus (apertural view); 25. Murex tribulus (lateral view); 25a. Murex tribulus (apertural view); 26. Semiricinula konkanensis (lateral view); 26a. Semiricinula konkanensis (apertural view); 27. Thais sp. (lateral view); 27a. Thais sp. (Apertural view); 28. Morula nodicostata (lateral view); 28a. Morula nodicostata (apertural view). 


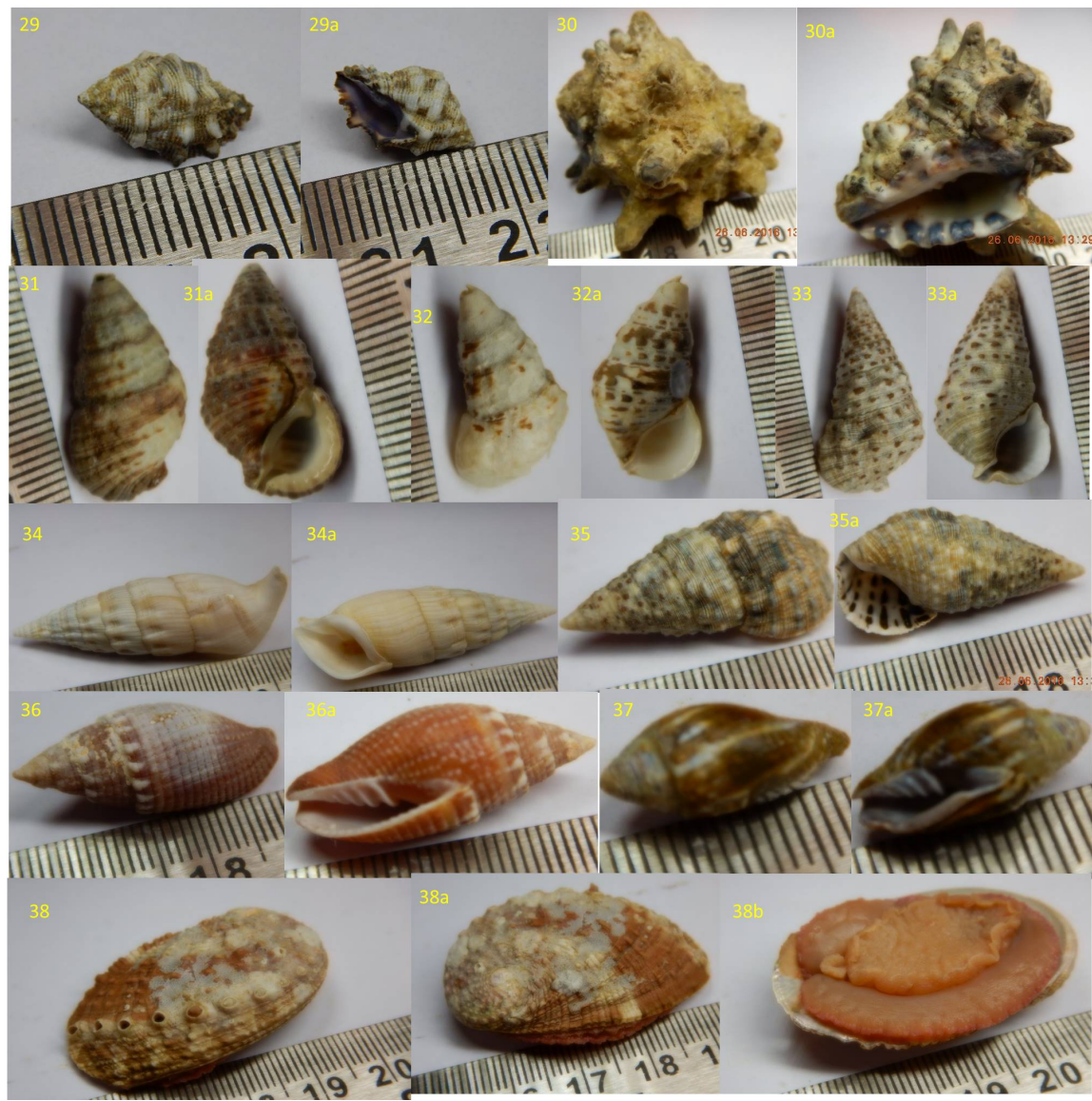

Figure A4. 29. Morula sp. (lateral view); 29a. Morula sp. (apertural view); 30. Vasum turbinellus (lateral view); 30. Vasum turbinellus (aperture view); 31. Clypeomorus bifasciata (lateral view); 31a. Clypeomorus bifasciata (ventral view); 32. Clypeomorus petrosa (lateral view); 32a. Clypeomorus petrosa (apertural view); 33. Certhium coralium (lateral view); 33a. Certhium coralium (apertural view); 34. Rhinochoclavis vertagus (lateral view); 34a. Rhinochoclavis vertagus (lateral view); 35. Batillaria multiformis (lateral view); 35a.Batillaria multiformis (apertural view); 36. Nebularia aurantia (lateral view); 36a. Nebularia aurantia (apertural view); 37. Strigatella paupercula (lateral view); 37a. Strigatella paupercula (apertural view); 38. Haliotis planata (lateral view); 38a. Haliotis planata (ventral view); 38b. Haliotis planta (apertural view). 


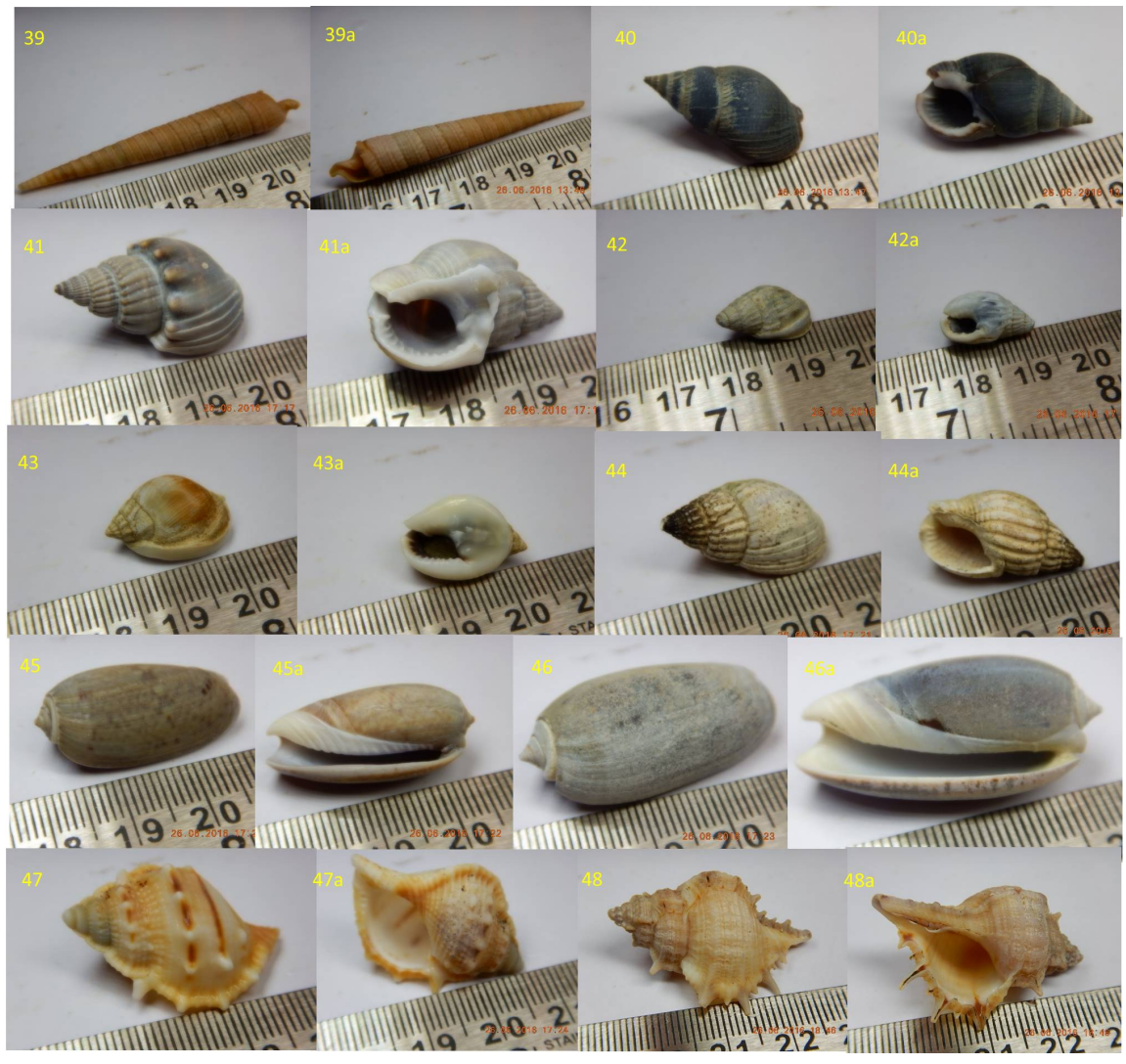

Figure A5. 39. Turritella sp. (lateral view); 39a. Turritella sp. (apertural view); 40. Nassarius sp. (lateral view); 40a. Nassarius sp. (apertural view); 41. Nassarius coronatus (lateral view); 41a. Nassarius coronatus (aperture view); 42. Nassarius olivaceus (lateral view); 42a. Nassarius olivaceus (apertural view); 43. Nassarius pullus (lateral view); 43a. Nassarius pullus (apertural view); 44. Nassarius conoidalis (lateral view); 44a. Nassarius conoidalis (apertural view); 45. Oliva vidua (lateral view); 45a. Oliva vidua (apertural view); 46. Oliva sp. (lateral view); 46a. Oliva sp (apertural view); 47. Bufonaria rana (lateral view); 47a. Bufonaria rana (apertural view); 48. Bufonaria echinata (lateral view); 48a. Bufonaria echinata (apertural view). 


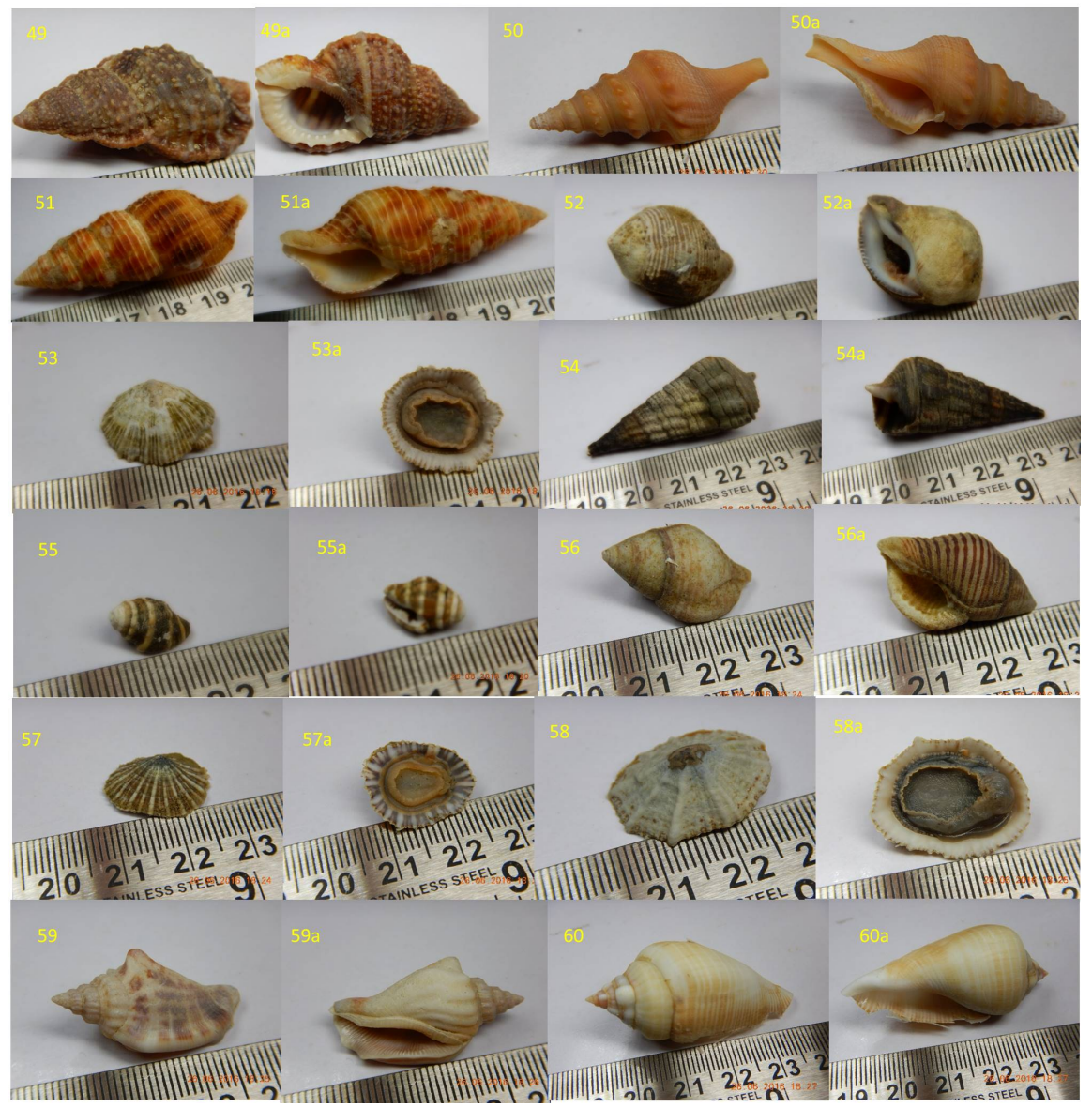

Figure A6. 49. Bursa granularis (lateral view); 49. Bursa granularis (apertural view); 50. Gammula vagata (lateral view); 50a.Gammula vagata (apertural view); 51. Turrilatirus craticulatus (lateral view); 51a. Turrilatirus craticulatus (apertural view); 52. Latirolagena smaragdulus (lateral view); 52a. Latirolagena smaragdulus (apertural view); 53. Scutellastra flexuosa (lateral view); 53a. Scutellastra flexuosa (lateral view); 54. Terebralia paluster (lateral view); 54a. Terebralia paluster (apertural view); 55. Engina mendicaria (lateral view); 55a. Engina mendicaria view); 57. Siphonaria sp1. (lateral view); 57a. Siphonaria sp1. (apertural view). 58. Siphonaria sp2. (lateral view); 58a. Siphonaria sp2. (apertural view); 59. Canarium labiatum (lateral view); 59a. Canarium labiatum (apertural view); 60. Gibberulus gibberulus (lateral view); 60a. Gibberulus gibberulus (apertural view). 


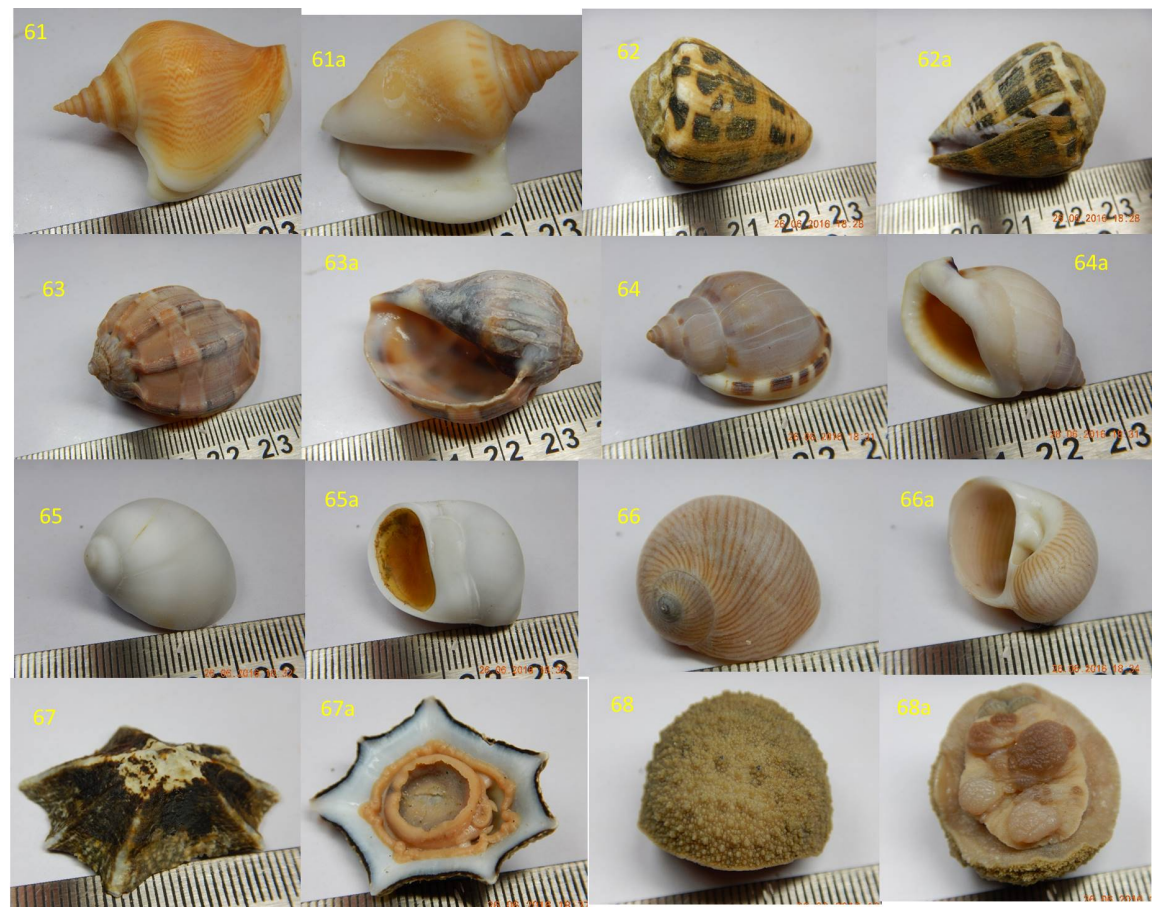

Figure A7. 61. Laevistrombus canarium (lateral view); 61a. Laevistrombus canarium (apertural view); 62. Conus ebraeus (lateral view); 62a, Conus ebraeus (apertural view); 63. Harpa major (lateral view); 63a. Harpa major (apertural view); 64. Semicassis bisulcata (lateral view); 64a. Semicassiss bisulcata (apertural view); 65. Polinices mammilla (lateral view); 65a. Polinices mammilla (apertural view); 66. Tanea lineata (lateral view); 66a. Tanea lineate (apertural view); 67. Patelloida saccharina (lateral view); 67a. Patelloida saccharina (apertural view); 68. Onchidium sp. (lateral view); 68a. Onchidium sp. (apertural view).

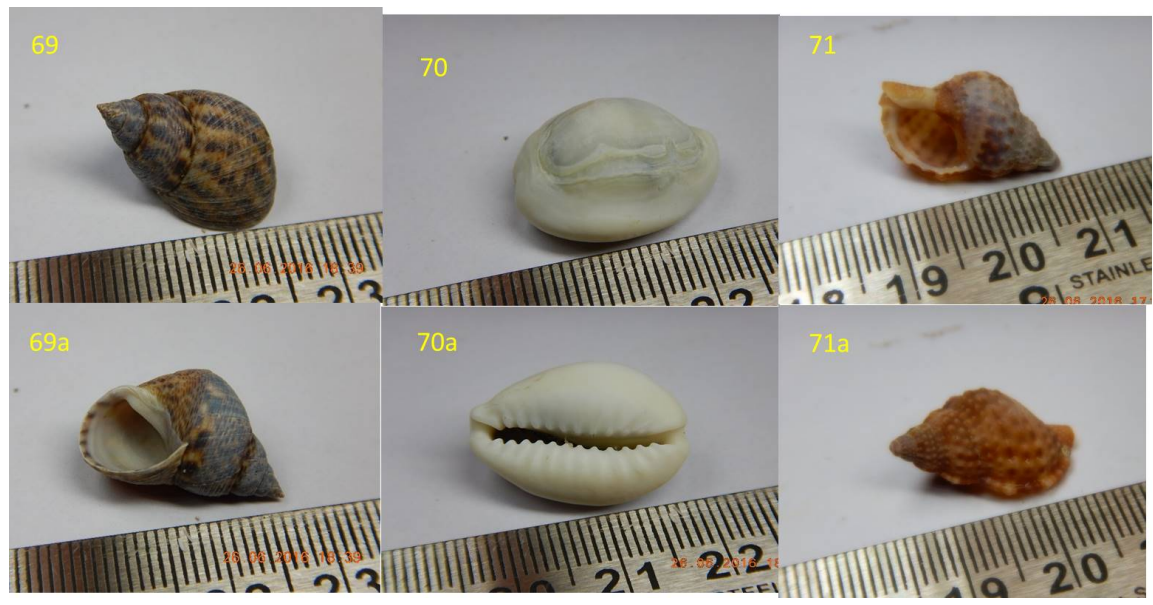

Figure A8. 69. Littoraria undulata (lateral view); 69a. Littoraria undulata (apertural view); 70. Monetaria moneta (lateral view); 70a. Monetaria moneta (apertural view); 71. Gyrineum natator (lateral view); 71a. Gyrineum natator (apertural view). 